\title{
Dynamical dessins are dense
}

\section{Christopher J. Bishop and Kevin M. Pilgrim}

\begin{abstract}
We apply a recent result of the first author to prove the following result: any continuum in the plane can be approximated arbitrarily closely in the Hausdorff topology by the Julia set of a postcritically finite polynomial with two finite postcritical points.
\end{abstract}

\section{Introduction}

Given compact subsets $A, B \subset \mathbb{C}$ their Hausdorff distance $d(A, B)$ is given by

$$
d(A, B):=\inf \left\{r: A \subset N_{r}(B), B \subset N_{r}(A)\right\}
$$

where $N_{r}(A), N_{r}(B)$ denote the $r$-neighborhoods of $A$ and $B$, respectively. Given a polynomial $g \in \mathbb{C}[z]$, we denote by $g^{j}$ the $j$ th iterate of $g$, and define its

- filled-in Julia set $K(g):=\left\{z: g^{j}(z) \nrightarrow \rightarrow \infty\right\}$, and

- Julia set $J(g):=\partial K(g)$.

K. Lindsey ([4], Theorem 2.2) has shown:

Theorem 1. Given any Jordan curve $\mathcal{J}$ bounding a closed topological disk $\mathcal{K}$ and any $\epsilon>0$, there exists a polynomial $g \in \mathbb{C}[z]$ such that

(1) $d(K(g), \mathcal{K})<\epsilon$,

(2) $d(J(g), \mathcal{J})<\epsilon$.

The proof is constructive; the above paper illustrates the result of applying the method of proof to a Jordan domain $\mathcal{K}$ outlining the figure of a cat, yielding a polynomial $g$ of degree 301 .

In this note, a continuum is a compact connected subset of $\mathbb{C}$. It is elementary to show that any continuum can be approximated arbitrarily closely in the Hausdorff topolology by a Jordan curve. Conclusion (2) of Theorem 1 then implies:

Mathematics Subject Classification (2010): Primary 37F10; Secondary 14H57, 37B45.

Keywords: Dessin, Julia set. 
Corollary 1. Given any continuum $K$ and any $\epsilon>0$, there exists a polynomial $g \in \mathbb{C}[z]$ such that $d(J(g), K)<\epsilon$.

In this note, we generalize Corollary 1.

Before stating our main result, we recall some definitions. A continuum is a dendrite if it is locally connected and has empty interior. Given a complex polynomial $p \in \mathbb{C}[z]$, a complex number $c$ is a critical point of $p$ if $p^{\prime}(c)=0$; its image $p(c)$ is a critical value. We denote by $C(p):=\left\{c: p^{\prime}(c)=0\right\}$ the set of critical points of $p$. A polynomial $f$ is a Belyi polynomial if $\operatorname{deg}(f)>1$ and if its set of critical values $f(C(f))$ is contained in the set $\{0,1\}$; these have been much studied from many points of view, see, e.g., [7]. We next introduce some dynamical notions. A polynomial $g \in \mathbb{C}[z]$ is postcritically finite if $P(g):=\left\{g^{j}(c): c \in C(g), j>0\right\}$ is finite. If $g$ is postcritically finite, the following facts are known (see, e.g., [5]): $J(g)$ is connected and locally connected, and is a dendrite if and only if no element of $C(g)$ is periodic. In [6], a Belyi polynomial $g$ is called an extra-clean dynamical Belyi polynomial ${ }^{1}$ if $P(g)=\{0,1\}, g(0)=g(1)=0$, and $g^{\prime}(0) \neq 0, g^{\prime}(1) \neq 0$; we denote the set of such polynomials by $X D B P$. Note that if $g \in X D B P$ then $J(g)$ is a dendrite. Theorem 3.6 in [3] implies that each $g \in X D B P$ is naturally a point on a zero-dimensional variety defined over $\mathbb{Q}$. It follows that if $g \in X D B P$ then the coefficients of $g$ lie in the field $\overline{\mathbb{Q}}$ of algebraic numbers. Two polynomials $g_{1}, g_{2}$ are conjugate as dynamical systems if there exists $A(z)=a z+b, a, b \in \mathbb{C}, a \neq 0$, such that $g_{2}=A \circ g_{1} \circ A^{-1}$. We denote by

$$
\mathcal{G}:=\left\{A \circ g \circ A^{-1}: A(z)=a z+b, a, b \in \overline{\mathbb{Q}}, a \neq 0, g \in X D B P\right\} \subset \overline{\mathbb{Q}}[z] .
$$

Since $\overline{\mathbb{Q}}[z]$ is countable, so is $\mathcal{G}$.

Our main result is:

Theorem 2. Given any continuum $K \subset \mathbb{C}$ and any $\epsilon>0$, there exists a polynomial $g \in \mathcal{G}$ with $d(J(g), K)<\epsilon$.

A key ingredient in our proof is an approximation result of the first author wherein continua are approximated by sets of the form $f^{-1}([0,1])$, where $f$ is a Belyi polynomial and $[0,1] \subset \mathbb{C}$ is the unit interval.

In this paragraph, we introduce some terminology and perspective related to Belyi polynomials; see [7]. We denote by $B P$ the set of Belyi polynomials. If $f \in B P$, its dessin is $D(f):=f^{-1}([0,1])$. By ibid. Lemma $3.4, D(f)$ is a tree with vertices $V(f):=f^{-1}(\{0,1\})$; an edge $e$ of $D(f)$ is the closure of a component of $f^{-1}((0,1))$. Thinking of $[0,1]$ as a tree with a single edge and with two vertices $v_{0}=0, v_{1}=1$, the map $f: D(f) \rightarrow[0,1]$ sends a closed edge $e$ of $D(f)$ homeomorphically to the edge $[0,1]$. Thus the valence of a vertex $\tilde{v}$ of $D(f)$, defined as the number of edges incident to $\tilde{v}$, coincides with the local degree $\operatorname{deg}(f, \tilde{v})$ of $f$ at $\tilde{v}$, defined as the multiplicity of the zero of the polynomial $z \mapsto f(z)-f(\tilde{v})$. A leaf of $D(f)$ is a vertex $\tilde{v}$ of valence 1 . Hence a vertex $\tilde{v}$ of $D(f)$ is a critical point of $f$ if and only if it is not a leaf.

\footnotetext{
${ }^{1}$ The adjective 'clean' is inherited from a technical symmetry-breaking condition commonly assumed in the theory of dessins d'enfants; see [7]. The modifier 'extra' refers to the additional condition $g(0)=g(1)=0$, and $g^{\prime}(0) \neq 0, g^{\prime}(1) \neq 0$.
} 
The approximation result we use is the following theorem.

Theorem 3. Given any continuum $K \subset \mathbb{C}$ and any $\epsilon>0$, there exists $f \in B P$ for which (i) $d(D(f), K)<\epsilon$, (ii) for each $\tilde{v} \in V(f), \operatorname{deg}(f, \tilde{v}) \leq 4$, and (iii) the coefficients of $f$ belong to $\overline{\mathbb{Q}}$.

Proof. Conclusion (i) is Theorem 1.1 in [2]; (ii) follows from its proof; see op. cit. $\S 3$, paragraph 3. We now prove (iii). Let $f \in B P$ satisfy (i) with $d(D(f), K)<\epsilon / 2$ and also (ii). Belyi's theorem and the Grothendieck correspondence [7] imply that there exists $h_{0}(z)=a_{0} z+b_{0}, a_{0}, b_{0} \in \mathbb{C}, a_{0} \neq 0$, for which $f \circ h_{0} \in \overline{\mathbb{Q}}[z]$. Using the density of $\overline{\mathbb{Q}}$ in $\mathbb{C}$, choose $a_{1}, b_{1} \in \overline{\mathbb{Q}}$ with $a_{1} \approx a_{0}, b_{1} \approx b_{0}$ so that

$$
\max \left\{\left|\left(h_{1} \circ h_{0}^{-1}\right)(z)-z\right|: z \in D(f)\right\}<\epsilon / 2,
$$

and put $f_{1}:=f \circ h_{0} \circ h_{1}^{-1} \in \overline{\mathbb{Q}}[z]$. Then $f_{1}$ satisfies conditions (ii) and (iii), and (i) holds since $D\left(f_{1}\right)=\left(h_{1} \circ h_{0}^{-1}\right)(D(f))$ and

$$
d\left(D\left(f_{1}\right), K\right) \leq d\left(D\left(f_{1}\right), D(f)\right)+d(D(f), K)<\epsilon .
$$

The proof of our main result, Theorem 2, has two steps. Suppose $K \subset \mathbb{C}$ is a continuum and $\epsilon>0$ is given.

(1) We apply Theorem 3 to obtain a polynomial $f \in B P \cap \overline{\mathbb{Q}}[z]$ satisfying both $d(D(f), K)<\epsilon / 2$ and the valence condition (ii).

(2) We define a sequence of polynomials $g_{n} \in \mathcal{G}$ such that $d\left(J\left(g_{n}\right), D(f)\right) \rightarrow 0$ as $n \rightarrow \infty$. The convergence will be proven in Lemma 1 ; it is here we use the valence condition on $f$. Then, choosing $n$ such that $d\left(J\left(g_{n}\right), D(f)\right)<\epsilon / 2$ will establish that $d\left(J\left(g_{n}\right), K\right)<\epsilon$, completing the proof.

In the next two paragraphs, we construct the polynomials $g_{n}$.

Let $q(z):=4 z(1-z)$. Note that $q \in B P$, that $q([0,1])=q^{-1}([0,1])=[0,1]$, and that $q(0)=q(1)=0$, with $C(q)=\{1 / 2\}$. For each $n \in \mathbb{N}, n \geq 1$, we have $q^{n} \circ f \in B P \cap \overline{\mathbb{Q}}[z]$ and $D\left(q^{n} \circ f\right)=D(f)$ as subsets of $\mathbb{C}$. Their tree structures differ: each edge of $D(f)$ is a union of $2^{n}$ edges of $D\left(q^{n} \circ f\right)$. It is easy to see that the set of leaves of $D\left(q^{n} \circ f\right)$ coincides with the set of leaves of $D(f)$, and that if $\tilde{v}$ is such a leaf then $\left(q^{n} \circ f\right)(\tilde{v})=0$. Lemma 2 will say that we can make edges of $q^{n} \circ f$ as small as we like by choosing $n$ sufficiently large. Since $D\left(q^{n} \circ f\right)=D(f)$ as sets, the valence of the tree $D\left(q^{n} \circ f\right)$ remains bounded above by 4 .

We now turn $q^{n} \circ f$ into a dynamical system; cf. [6]. Suppose $v_{0}, v_{1} \in V(f)$ are leaves of $D(f)$, that is, vertices of valence 1. By replacing $f$ with $q \circ f$, we may assume that $f\left(v_{0}\right)=f\left(v_{1}\right)=0$. The assumption $f \in \overline{\mathbb{Q}}[z]$ implies $v_{0}, v_{1} \in \overline{\mathbb{Q}}$. Let $A(z)=\left(v_{1}-v_{0}\right) z+v_{0}$, so that $A(0)=v_{0}, A(1)=v_{1}$. Fix $n \in \mathbb{N}$. Let $g_{n}:=A \circ q^{n} \circ f$.

The paragraph below discusses the properties of the polynomials $g_{n}$.

By construction, $g_{n} \in \overline{\mathbb{Q}}[z]$ and $g_{n}$ has two critical values, namely $v_{0}$ and $v_{1}$. We have $D(f)=D\left(q^{n} \circ f\right)=g_{n}^{-1}\left(\left[v_{0}, v_{1}\right]\right)$ as sets. As trees, now an edge $e$ of $D\left(q^{n} \circ f\right)$ is the closure of a component of $g_{n}^{-1}\left(\left(v_{0}, v_{1}\right)\right)$, where $\left(v_{0}, v_{1}\right)$ is the interval $\left[v_{0}, v_{1}\right]$ minus its endpoints. Abusing notation slightly, we denote by $V\left(g_{n}\right):=$ $g_{n}^{-1}\left(\left\{v_{0}, v_{1}\right\}\right)$ the set of vertices of $D\left(q^{n} \circ f\right)$. Each critical point of $g_{n}$ maps under $g_{n}$ 
either to $v_{0}$ or to $v_{1}$; by construction, $v_{0}=g_{n}\left(v_{0}\right)=g_{n}\left(v_{1}\right)$, and $g_{n}^{\prime}\left(v_{0}\right) \neq 0$, $g_{n}^{\prime}\left(v_{1}\right) \neq 0$. It follows that $P\left(g_{n}\right)=\left\{v_{0}, v_{1}\right\} \subset \overline{\mathbb{Q}}$, so that $g_{n}$ is postcritically finite, and that every critical point lands on the fixed point $v_{0}$ under iteration of $g_{n}$. It is a general fact that all fixed points of a postcritically finite map $g_{n}$ are either critical points or they lie in the Julia set. We conclude $v_{0} \in J\left(g_{n}\right)$. Since $g_{n}\left(v_{1}\right)=v_{0}$, we have $v_{1} \in J\left(g_{n}\right)$ too. Hence $V\left(g_{n}\right)=g_{n}^{-1}\left(\left\{v_{0}, v_{1}\right\}\right) \subset J\left(g_{n}\right)$ by invariance of $J\left(g_{n}\right)$; moreover, $J\left(g_{n}\right)$ is a dendrite. The valence condition on $f$ implies that the local degree of $g_{n}$ at any point is at most 4. Since $A^{-1} \circ g_{n} \circ A \in X D B P$ and $A \in \overline{\mathbb{Q}}[z]$, we conclude $g_{n} \in \mathcal{G}$.
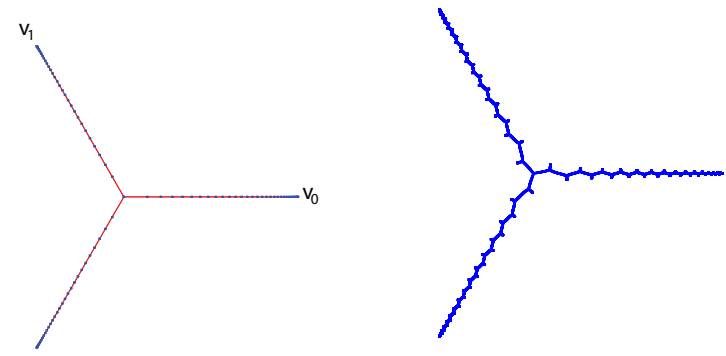

Figure 1. At left: the $\operatorname{dessin} D\left(q^{5} \circ f\right)=D(f)$ where $f(z)=z^{3}$, with leaves $v_{0}, v_{1}$ marked. At right: an approximation of $J\left(g_{5}\right)$ by the set $g_{5}^{-1}(D(f))$; its greater apparent thickness is an artifact of plotting the $3^{2} \cdot 2^{10}-1$ preimages of the vertices of $D(f)$. Images courtesy of Don Marshall.

The proof of Theorem 2 then rests upon establishing the closeness that Figure 1 suggests:

Lemma 1. The Hausdorff distance $d\left(J\left(g_{n}\right), D(f)\right) \rightarrow 0$ as $n \rightarrow \infty$.

\section{Proof of Lemma 1}

Suppose $f, q, n, g_{n}$ are as in step 2 of the outline given in the Introduction.

Lemma 2. The maximum diameter of an edge e of $D\left(q^{n} \circ f\right)$ tends to zero as $n \rightarrow \infty$.

Proof. An easy exercise shows the conclusion holds when $f=q$. Now suppose $f \in B P$. Since the inverse branches of $f$ are uniformly continuous on $(0,1)$, the general conclusion holds.

Let $D:=D(f)$. We recall from step 2 the following: $D=g_{n}^{-1}\left(\left[v_{0}, v_{1}\right]\right)$; the set $g_{n}^{-1}\left(\left\{v_{0}, v_{1}\right\}\right)$ is the set of vertices of the tree $D$; the edges of $D$ are the closures of the components of $g_{n}^{-1}\left(v_{0}, v_{1}\right)$, where $\left(v_{0}, v_{1}\right)$ is the Euclidean segment $\left[v_{0}, v_{1}\right]$ minus its endpoints.

We are going to cover $D$ by a certain pair of Jordan domains $W_{i}$ with the property that $W_{i} \cap\left\{v_{0}, v_{1}\right\}=v_{i}, i=0,1$. See Figure 2 . 


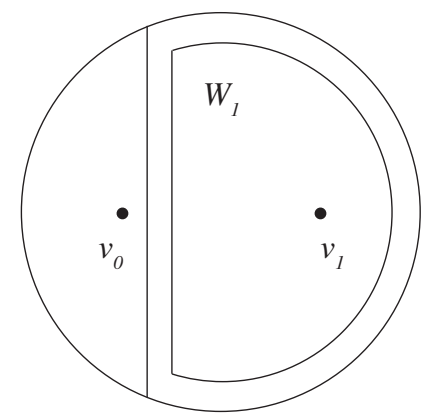

Figure 2. Caricature of $W_{1}$. The domain $W_{0}$ is similar. The disk shown is $B:=B\left(\frac{v_{1}+v_{0}}{2}, 10 M\right)$. The domain $\widehat{W}_{1}$ is the portion of the disk to the right of the longer vertical segment. The figure is not to scale; one should imagine that $v_{0}, v_{1}$ appear much closer together compared to the diameter of $B$, and that $D$ is contained in the smaller disk $\frac{1}{10} B$ with the same center and $\frac{1}{10}$ th the radius.

Their precise definition is a bit technical; we will give it later. Let $W$ denote either of the domains $W_{0}, W_{1}$, and let $\widetilde{W}$ be a connected component of $g_{n}^{-1}(W)$; it will also be a Jordan domain. We will show diam $\widetilde{W} \rightarrow 0$ uniformly in $n$ (Lemma 3). Lemma 1 will then follow easily.

In order to control the diameters of the domains $\widetilde{W}$, we will thicken the domains $W_{0}, W_{1}$ to Jordan domains $\widehat{W}_{0}, \widehat{W}_{1}$ so that $\bar{W}_{i} \subset \widehat{W}_{i}$ and in addition $\widehat{W}_{i} \cap\left\{v_{0}, v_{1}\right\}=$ $W_{i} \cap\left\{v_{0}, v_{1}\right\}=v_{i}, i=0,1$. Now suppose $W, \widetilde{W}$ are as in the previous paragraph. Let $\widehat{W}$ be the thickening of $W$. There is a unique component $\widetilde{\widehat{W}}$ of $g_{n}^{-1}(\widehat{W})$ that contains $\widetilde{W}$; it is a thickening of $\widetilde{W}$. The "Koebe space" $\widetilde{\widetilde{W}} \backslash \overline{\widetilde{W}}$ will allow us to control distortion and relate the diameter of $\widetilde{W}$ to the diameter of the edge it meets.

Suppose $W, \widetilde{W}, \widetilde{W}, \widetilde{\widehat{W}}$ are as in the previous two paragraphs. Choose a point $v:=W \cap\left\{v_{0}, v_{1}\right\}$; it is a branch value of $g_{n}$. Since $g_{n}$ is a polynomial, we obtain a map of pairs $g_{n}:(\widetilde{\widehat{W}}, \widetilde{W}) \rightarrow(\widehat{W}, W)$ in which each restriction is proper and each domain is a Jordan domain. Since $\widehat{W}$ contains exactly one branch value of $g_{n}$, the preimage $\widetilde{\widetilde{W}} \cap g_{n}^{-1}(v)$ consists of a single point, which we will denote by $\tilde{v}$, which is a vertex of $D$. Since $v \in W$, we have $\tilde{v} \in \widetilde{W}$. Let $k:=\operatorname{deg}\left(g_{n}, \tilde{v}\right)$. Since the ramification of $g_{n}: \widetilde{\widehat{W}} \rightarrow \widehat{W}$, if there is any, occurs at the unique point $\tilde{v}$, we have $\operatorname{deg}\left(g_{n}: \widetilde{\widehat{W}} \rightarrow \widehat{W}\right)=k$ as well. The control on the local degrees of the polynomial $f$ in Theorem 3 shows that $k \leq 4$. Let $\mathbb{D}$ denote the open unit disk in $\mathbb{C}$. Up to precomposition with a rotation about the origin, there exists a unique Riemann map $\phi:(\mathbb{D}, 0) \rightarrow(\widehat{W}, v)$. Since $g_{n}: \widetilde{\widehat{W}} \rightarrow \widehat{W}$ is ramified only possibly at $\tilde{v}$, we obtain a Riemann map $\widetilde{\phi}:(\mathbb{D}, 0) \rightarrow(\widetilde{\widehat{W}}, \tilde{v})$ such that the following diagram 
commutes:

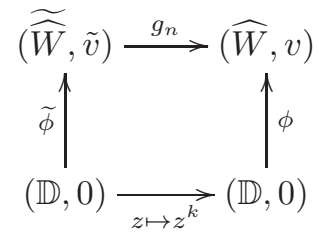

We will apply the Koebe distortion principle to the map $\widetilde{\phi}$ and conclude that the diameter of $\widetilde{W}$ is bounded from above in terms of the diameters of the edges of $D$; by Lemma 2 , these tend to zero as $n \rightarrow \infty$.

We now construct the domains $W_{0}, W_{1}$. First, denote $M:=\operatorname{diam}(D)$ and $B(a, r):=\{z \in \mathbb{C}:|z-a|<r\}$. Next see Figure 2 .

We now give the definitions of the sets $W_{i}$ and $\widehat{W}_{i}$. Let

$$
\begin{aligned}
v_{0}^{\prime} & :=\frac{7 v_{0}+v_{1}}{8}, v_{0}^{\prime \prime}=\frac{3 v_{0}+v_{1}}{4} \\
v_{1}^{\prime} & :=\frac{v_{0}+7 v_{1}}{8}, v_{1}^{\prime \prime}=\frac{v_{0}+3 v_{1}}{4} \\
\widehat{W}_{1-i} & :=B\left(\frac{v_{1}+v_{0}}{2}, 10 M\right) \cap\left\{\left|z-v_{i}^{\prime}\right|<\left|z-v_{i}\right|\right\}, i=0,1 \\
W_{1-i} & :=B\left(\frac{v_{0}+v_{1}}{2}, 9 M\right) \cap\left\{\left|z-v_{i}^{\prime \prime}\right|<\left|z-v_{i}\right|\right\}, i=0,1 .
\end{aligned}
$$

By construction,

- $\widehat{W}_{i} \cap\left\{v_{0}, v_{1}\right\}=W \cap\left\{v_{0}, v_{1}\right\}=v_{i}, i=0,1$;

- $D \subset W_{0} \cup W_{1}$;

- $\widehat{W}_{i} \backslash \overline{W_{i}}$ is an annulus, $i=0,1$.

Lemma 3. The maximum diameter of a component $\widetilde{W}$ tends to zero as $n \rightarrow \infty$.

Proof. Suppose $g_{n}:(\widetilde{\widehat{W}}, \widetilde{W}) \rightarrow(\widehat{W}, W)$ is a map of pairs as in the preceding paragraphs; we adopt the notation used there. Up to precomposition with rotations about the origin, the map $\phi$ is one of only two possible Riemann maps. Hence there exist $0<r<s<1$ such that if $U:=\phi^{-1}(W)$, then

$$
B(0, r) \subset U \subset B(0, s) \subset \mathbb{D} .
$$

Denote

$$
\widetilde{U}:=\left\{z \in \mathbb{D} \mid z^{k} \in U\right\} .
$$

From the second part of Theorem 3 we have $1 \leq k \leq 4$. Hence

$$
r \leq \tilde{r}:=r^{1 / k}, \quad \tilde{s}:=s^{1 / k} \leq s^{1 / 4},
$$

and

$$
B(0, r) \subset B(0, \tilde{r}) \subset \widetilde{U} \subset B(0, \tilde{s}) \subset B\left(0, s^{1 / 4}\right) \subset \mathbb{D}
$$


note that $r$ and $s^{1 / 4}$ do not depend on the choice of component $\widetilde{\widehat{W}}$. By definition, the following diagram commutes:

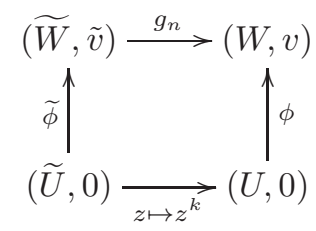

The rescaled map $\psi:=\left|\widetilde{\phi}^{\prime}(0)\right|^{-1}(\widetilde{\phi}-\widetilde{\phi}(0))$ is an element of the class of so-called Schlicht functions: injective holomorphic maps $\psi: \mathbb{D} \rightarrow \mathbb{C}$ with the normalization $\psi(0)=0, \psi^{\prime}(0)=1$. By Theorem 5.3 in [1], for all $z \in \mathbb{D}$ and all Schlicht functions $\psi$,

$$
|z|(1+|z|)^{-2} \leq|\psi(z)| \leq|z|(1-|z|)^{-2} .
$$

Hence upon setting

$$
\rho:=r(1+r)^{-2}, \quad \sigma:=s^{1 / 4}\left(1-s^{1 / 4}\right)^{-2}, \quad \delta:=\left|\widetilde{\phi}^{\prime}(0)\right|
$$

we have by (2.1) that

$$
B(\tilde{v}, \rho \delta) \subset \widetilde{\phi}(\widetilde{U})=\widetilde{W} \subset B(\tilde{v}, \sigma \delta) .
$$

Let $e$ be any one of the $k$ components of $g_{n}^{-1}\left(\left(v_{0}, v_{1}\right)\right)$ whose closure meets $\tilde{v}$; the closure of $e$ is an edge of $D$ containing $\tilde{v}$. Since $\left(v_{0}, v_{1}\right) \not \subset W$, we have $e \not \subset \widetilde{W}$, so

$$
\rho \delta<\operatorname{diam}(e)
$$

which implies

$$
\sigma \delta<\operatorname{diam}(e) \frac{\sigma}{\rho}
$$

and so

$$
\operatorname{diam}(\widetilde{W}) \leq 2 \sigma \delta<2 \operatorname{diam}(e) \frac{\sigma}{\rho} \rightarrow 0
$$

as $n \rightarrow \infty$, by Lemma 2 . The constants $\rho, \sigma$ are independent of $n$ and of the choice of $\tilde{v}$, so the proof of Lemma 3 is complete.

Proof of Lemma 1. Let $W_{0}, W_{1}$ be the domains as defined above, and let $\widetilde{W}_{\tilde{v}}$, $\tilde{v} \in V:=g_{n}^{-1}\left(\left\{v_{0}, v_{1}\right\}\right)$ denote the components of preimages $g_{n}^{-1}\left(W_{i}\right), i \in\{0,1\}$. Denote $J:=J\left(g_{n}\right)$. Pick $\epsilon<\frac{1}{2} \inf \left\{|a-b|: a \in D, b \in \mathbb{C} \backslash \overline{W_{0} \cup W_{1}}\right\}$. Apply Lemma 3 to obtain $n$ so that $\operatorname{diam}\left(\widetilde{W}_{\tilde{v}}\right)<\epsilon$ for all $\tilde{v} \in V\left(g_{n}\right)$. Each $\widetilde{W}_{\tilde{v}}$ is a Jordan domains, so it has the same diameter as its closure.

On the one hand, by our choice of $\epsilon$,

$$
g_{n}^{-1}\left(\overline{W_{0} \cup W_{1}}\right)=\bigcup_{\tilde{v} \in V} \overline{\widetilde{W}_{\tilde{v}}} \underbrace{\subset}_{\text {Lemma } 3} N_{\epsilon}(D) \subset \overline{W_{0} \cup W_{1}}
$$

and so $\overline{W_{0} \cup W_{1}}$ is backward-invariant under $g_{n}$. It is a general fact that $J$ may be equivalently defined as the smallest closed subset of $\mathbb{C}$ satisfying $\# J>1$ and $g_{n}^{-1}(J) \subset J ;$ see $[5]$. 
Thus $J \subset \overline{W_{0} \cup W_{1}}$. By invariance of $J$ we have then

$$
J \subset g_{n}^{-1}\left(\overline{W_{0} \cup W_{1}}\right)=\bigcup_{\tilde{v} \in V} \overline{\widetilde{W}_{\tilde{v}}} \subset N_{\epsilon}(D) .
$$

On the other hand, recalling the last sentence of Step 2, we have $V \subset J$, and $\left[v_{0}, v_{1}\right] \subset W_{0} \cup W_{1}$ implies $D=g_{n}^{-1}\left(\left[v_{0}, v_{1}\right]\right) \subset g_{n}^{-1}\left(W_{0} \cup W_{1}\right)=\bigcup_{\tilde{v} \in V} \widetilde{W}_{\tilde{v}}$, so by our choice of $\epsilon$ and $n$, we have

$$
N_{\epsilon}(J) \supset N_{\epsilon}(V) \supset \bigcup_{\tilde{v} \in V} \widetilde{W}_{\tilde{v}} \supset D .
$$

This completes the proof of Lemma 1 and establishes Theorem 2.

\section{References}

[1] Ahlfors, L. V.: Conformal invariants: topics in geometric function theory. McGraw-Hill Series in Higher Mathematics, McGraw-Hill, New York-DüsseldorfJohannesburg, 1973.

[2] Bishop, C. J.: True trees are dense. Invent. Math. 197 (2014), no. 2, 433-452.

[3] Brezin, E., Byrne, R., Levy, J., Pilgrim, K. And Plummer, K.: A census of rational maps. Conform. Geom. Dyn. 4 (2000), 35-74.

[4] Lindsey, K. A.: Shapes of polynomial Julia sets. Ergodic Theory Dynam. Systems 35 (2015), no. 6, 1913-1924.

[5] Milnor, J.: Dynamics in one complex variable. Third edition. Annals of Mathematics Studies 160, Princeton University Press, NJ, 2006.

[6] Pilgrim, K. M.: Dessins d'enfants and Hubbard trees. Ann. Sci. École Norm. Sup. (4) 33 (2000), 671-693.

[7] Schneps, L.: Dessins d'enfants on the Riemann sphere. In The Grothendieck theory of dessins d'enfants (Luminy, 1993), 47-77. London Math. Soc. Lecture Note Ser. 200, Cambridge Univ. Press, Cambridge, 1994.

Received October 1, 2013; revised February 6, 2014.

Christopher J. Bishop: Department of Mathematics, Stony Brook University, Stony Brook, NY 11794-3651 USA.

E-mail: bishop@math.sunysb.edu

Kevin M. PILgRim: Department of Mathematics, Indiana University, Bloomington, IN 47405-7106, USA.

E-mail: pilgrim@indiana.edu

The first author was supported by NSF grant DMS 130-5233. The second author was supported by Simons foundation grant 245269. This collaboration arose during the Institute for Pure and Applied Mathematics' program Interactions Between Analysis and Geometry, Dynamics of Groups and Rational Maps workshop; we thank IPAM for its hospitality. The authors thank the referees for helpful comments. 